\title{
Calculation of Sensitivities in Thermal Control Systems with Nonlinear Inequality Constraints
}

\author{
S. Suresha* \\ Indian Space Research Organisation, Bangalore 560017, India \\ and \\ S. C. Gupta ${ }^{\dagger}$ \\ Indian Institute of Science, Bangalore 560012, India
}

\begin{abstract}
The design of a thermal system is studied using temperature sensitivities. The thermal system is subjected to nonlinear temperature constraints, which are in the form of inequalities. Temperature sensitivities and the derivatives of the temperature constraints with respect to different design parameters are calculated by different methods, and their relative computational efforts are discussed. The thermal system pertaining to a shunt regulator in a spacecraft is considered. For thermal sensitivity analysis, design parameters such as internal heat dissipation, optical properties of an optical solar reflector, emittance of black paint, and emittance of low-emittance tape are considered. The numerical results indicate that the absorptance of the optical solar reflector is the most important parameter in the thermal control of a shunt regulator. An appropriate thermal shield and an increase in the black painted area are suggested as suitable design strategies to control the temperature of the shunt regulator.
\end{abstract}

\section{Nomenclature}

$=$ total surface area of the nodal element exposed to solar radiation, $\mathrm{m}^{2}$

$=$ cross-sectional area of the nodal element, $\mathrm{m}^{2}$

$=$ surface area of the nodal element, $\mathrm{m}^{2}$

$=$ matrix representing the temperature sensitivity functions as defined in Eq. (19), K/unit of the parameter considered

$=$ matrix having components $\partial f_{i} / \partial \boldsymbol{\beta}_{j}$, $i=1,2, \ldots, N, j=1,2, \ldots, M$, as defined in Eq. (19), W/unit of the parameter considered

$=$ geometrical view factor between the $i$ th and $j$ th nodes

= function defined in Eq. (5), W

$=$ temperature constraint function for the $i$ th node as defined in Eq. (6), K

$=$ heat generated at the $i$ th node, $\mathrm{W}$

$=$ thermal conductivity of the material in the $x_{i}$ direction, $i=1,2,3$, W/m K

$=$ linear elemental length between $i$ th and $j$ th nodes, $\mathrm{m}$

$=$ total number of design parameters considered

$=$ total number of nodes in the system

= row matrix as defined in Eq. (21)

$=$ solar intensity, $\mathrm{W} / \mathrm{m}^{2}$

$=$ temperature of the $i$ th element or node, $\mathrm{K}$

$=$ Cartesian coordinates of a point in space, $\mathrm{m}$

$=$ solar absorptance of the surface

$=j$ th design parameter

$=$ nominal value of the design parameter $\boldsymbol{\beta}_{j}$

$=$ variation of the temperature of the $i$ th node, $\mathrm{K}$

$=$ arbitrary small variation in the design parameter $\boldsymbol{\beta}_{j}$

$=$ infrared emittance of the surface

$=$ angle between surface normal and the sun vector

$=$ Stefan-Boltzmann constant, $\mathrm{W} / \mathrm{m}^{2} \mathrm{~K}^{4}$

$=$ vector as defined in Eq. (23)
Received Aug. 19, 1997; revision received March 10, 1998; accepted for publication March 30, 1998. Copyright (C) 1998 by the American Institute of Aeronautics and Astronautics, Inc. All rights reserved.

* Scientist, Thermal Systems Group, Satellite Centre.

${ }^{\dagger}$ Professor, Department of Mathematics.

$\begin{array}{ll}\text { Subscripts } & \\ i_{1, j, k} & =i \text { th, } j \text { th }, \text { and } k \text { th nodes } \\ l_{1} m & =l \text { th and } m \text { th elements } \\ \mathrm{sp} & =\text { space }\end{array}$

Superscripts

$T$

$-1$

$=$ transpose of a matrix

$=$ inverse of a matrix

\section{Introduction}

$\mathbf{C}$ ONTROL of high heat dissipation within electronic packages and the external heat loads on them from the surroundings is one of the major concerns of a thermal designer inasmuch as the temperatures of the components of the electronic packages are to be maintained in a specified range. This specified temperature range, when transformed into a mathematical model, results in nonlinear inequality constraintsfor temperatures. The present study pertains to a shunt regulator in a spacecraft system. The objective is to suggest an optimal design for the shunt regulatorby taking into consideration the variations in the design parameter values, which in turn change the temperatures.

Thermal sensitivity studies of spacecraft systems, as well as some other structures, have been the subject of many investigations. They are essentially of two types: In the first, the temperature sensitivities have been calculated without temperatures being subjected to constraints, whereas in the second, thermal sensitivity studies have been conducted incorporating the constraints imposed on the system. Goble ${ }^{1}$ discussed the steady-state temperature sensitivities associated with a Skylab orbital assembly without considering the constraints on temperatures. Similar studies have been conducted by Suresha et al. ${ }^{2}$ in connection with a transient problem concerning a spacecraft battery.

Haftka ${ }^{3}$ described the numerical techniques for computing the sensitivities of temperatures when the system is subjected to linear constraints. Haftka ${ }^{4}$ suggested a method to improve the accuracy of the calculated sensitivities. Haftka and Malkus ${ }^{5}$ considered large thermal systems with thousands of degrees of freedom and suggested ways of computing thermal sensitivities. They also discussed methods to reduce computational cost and errors in sensitivity calculations. Belegundu ${ }^{6}$ discussed design sensitivity analysis of thermal systems with constraints. The adjoint variable method and Lagrangian approach were considered, and it was pointed out that the final equations obtained from the Lagrangian approach are 